\title{
Solitary Waves in a Peridynamic Elastic Solid
}

\author{
Stewart A. Silling \\ Multiscale Science Department \\ Sandia National Laboratories \\ P.O Box 5800 \\ Albuquerque, NM 87185-1322
}

June 3, 2016

\begin{abstract}
The propagation of large amplitude nonlinear waves in a peridynamic solid is analyzed. With an elastic material model that hardens in compression, sufficiently large wave pulses propagate as solitary waves whose velocity can far exceed the linear wave speed. In spite of their large velocity and amplitude, these waves leave the material they pass through with no net change in velocity and stress. They are nondissipative and nondispersive, and they travel unchanged over large distances. An approximate solution for solitary waves is derived that reproduces the main features of these waves observed in computational simulations. It is demonstrated by numerical studies that the waves interact only weakly with each other when they collide. Wavetrains composed of many non-interacting solitary waves are found to form and propagate under certain boundary and initial conditions.
\end{abstract}

\section{Introduction}

A solitary wave is a wave that travels as a single pulse without dispersing or changing shape over time. Unlike a shock, which is another type of wave pulse, a solitary wave leaves the medium it passes through unchanged. Solitary waves have important applications in many areas of science including solid state physics, plasma physics, and water waves. Wave motion in loose 
granular media with nonlinear contact forces can result in solitary waves [9], and the characterization of solitary waves in heterogeneous solids is an area of active research [6]. Solitary waves are inherently nonlinear. Their stability results from the equilibrium between nonlinearities in the underlying physics that tend to make the pulse narrower and dispersive mechanisms that tend to make it wider. Although many authors have studied solitary waves in nonlinear one-dimensional lattices, especially the Toda lattice [16], little work has been done on these waves in nonlocal continuum mechanics models. A general discussion of solitary waves can be found in [19].

To see the relevance of solitary waves in solid mechanics, it is helpful to consider the simple example illustrated in Figure 1. A long, one-dimensional row of nearly rigid elastic beads has a constant gap spacing. The bead on the left is given an initial particle velocity. This velocity is imparted to its neighbor on the right when they make contact. A wave with constant velocity $V$ moves through the array as each successive pair of beads contacts each other. The wave velocity depends on the particle velocity, the gap spacing, the bead diameter, and the bead mass.

This simple example illustrates the essential features of solitary waves. As the wave moves, the material behind it is left unchanged (except for a rigid translation) since the gap distance remains the same. The wave velocity is not strongly dependent on the elastic properties of the beads. The mechanics underlying the wave is strongly nonlinear, since it depends on contact. The connection with peridynamics is that the mechanics of the problem is nonlocal, since it contains length scales (bead diameter and gap spacing) that strongly influence the dynamics. The importance of length scales in this example suggests that peridynamics, as a nonlocal theory, may be a natural setting in which to study the phenomenon of solitary waves. The results of the present paper show that, indeed, solitary wave solutions can be obtained for elastic peridynamic continua.

\section{Peridynamics background}

The peridynamic theory of continuum mechanics has been proposed as a generalization of the standard theory. It treats material defects and longrange forces within its basic field equations [11]. The essential feature of the theory is that material points separated by a finite distance can interact with each other directly through a material model. The theory is therefore 


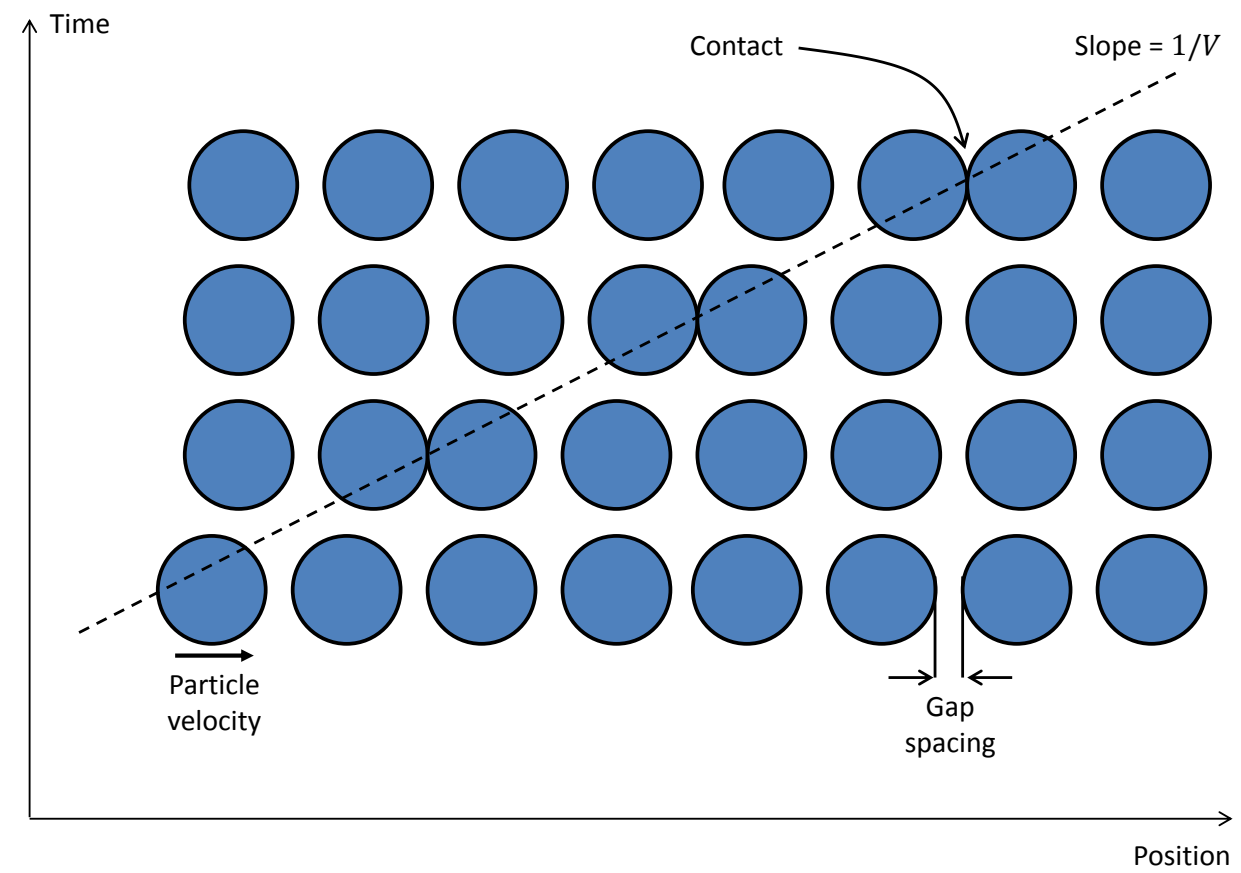

Figure 1: Simple example of solitary wave motion in mechanics: a string of beads with constant diameter, gap spacing, and mass. 
inherently nonlocal. The interaction between two material points is called a bond interaction.

The peridynamic equation of motion is given in one dimension by

$$
\rho u_{t t}(x, t)=\int_{-\delta}^{\delta} f(u(x+\xi, t)-u(x, t), \xi) d \xi+b(x, t)
$$

where $\rho$ is the reference density (assumed constant), $u$ is the displacement field, and $b$ is the external body force density field. $\xi$ is the relative position between two interacting material points and is called a bond. $\delta$ is a positive distance called the horizon that represents a cutoff distance for the nonlocal interactions between material points. The function $f$ is the pairwise bond force density that contains the force interactions and is determined by the material model. For any material model, this function is required to possess the following anti-symmetry:

$$
f(-\eta,-\xi)=-f(\eta, \xi)
$$

for all $\xi$ and $\eta$. With this requirement, the equation of motion (1) satisfies conservation of linear momentum.

The form of $f$ stated above assumes that the force in a bond $\xi$ depends only on the relative displacement between the bond's endpoints. Material models that use this assumption are called bond-based. A more general treatment, which is not used in the remainder of this paper, is called state-based. It allows the force in a given bond also to depend on the relative displacement of other bonds [14].

Most applications of peridynamics use it for modeling damage and fracture. The form of (1), since it does not involve partial derivatives with respect to position, is compatible with discontinuities such as cracks. For applications involving damage and cracking, a damage evolution law is included with the material model that causes bonds to break irreversibly according to some damage criterion. The capability of peridynamics to model fracture within the basic field equations without the use of supplemental kinetic relations for crack evolution is sometimes called "unguided crack growth." Examples of dynamic fracture modeling with peridynamics can be found in [7].

Aside from fracture, the main capability provided by peridynamics is the incorporation of long-range forces as part of a material model. This provides a convenient and consistent way to treat nanoscale forces such as Van der Waals forces that involve a finite length scale for interactions [13]. In the 
present work, a particular nonlinear material model is used that qualitatively reproduces the effects of nonlinear contact and hardening under compression. Further details on the peridynamic theory, including a formulation of nonlocal thermodynamics that is consistent with the mechanical theory, can be found in the review article by Silling and Lehoucq [15] and the book by Madenci and Oterkus [8].

Waves in a peridynamic medium, including linear waves, are in general dispersive [11]. Dispersion results from the dependence of wave speed on wavelength. In the peridynamic model, this dependence results from the characteristic length scale provided by the horizon. Dispersion curves can be used to help determine the material model for a real material, including the appropriate value of the horizon [18]. Dispersion should not be confused with dissipation, which is the irreversible loss of mechanical energy from the system. The distinction will be important in the discussion of shocks in Section 5.2 later in this paper.

In the present paper, we analyze the propagation of special nondispersive waves in a nonlinear peridynamic solid. The existence of such waves is perhaps surprising because of the prevalence of dispersivity in linear peridynamic waves. In these nondispersive waves, a single pulse called a solitary wave propagates without change in shape and without energy loss over long distances at a velocity that exceeds the speed of linear waves (at any wavelength).

Figure 2 illustrates the qualitative difference between dispersive linear waves and solitary waves that can arise in a nonlinear peridynamic material. The left side of a bar is subjected to a jump in displacement of $0.0008 \mathrm{~m}$ at $t=0$. The linear material model predicts a complex wave pattern due to the dispersive nature of waves in a linear elastic peridynamic material. The nonlinear material model (to be described in the next section), whose elastic modulus is identical to that used in the linear model, predicts two wavefronts of small but finite thickness that propagate without dispersion. The velocity of these wavefronts is much greater than that of the linear waves. Because this type of wave propagates forever without any change in shape, it is called a "solitary wave." 


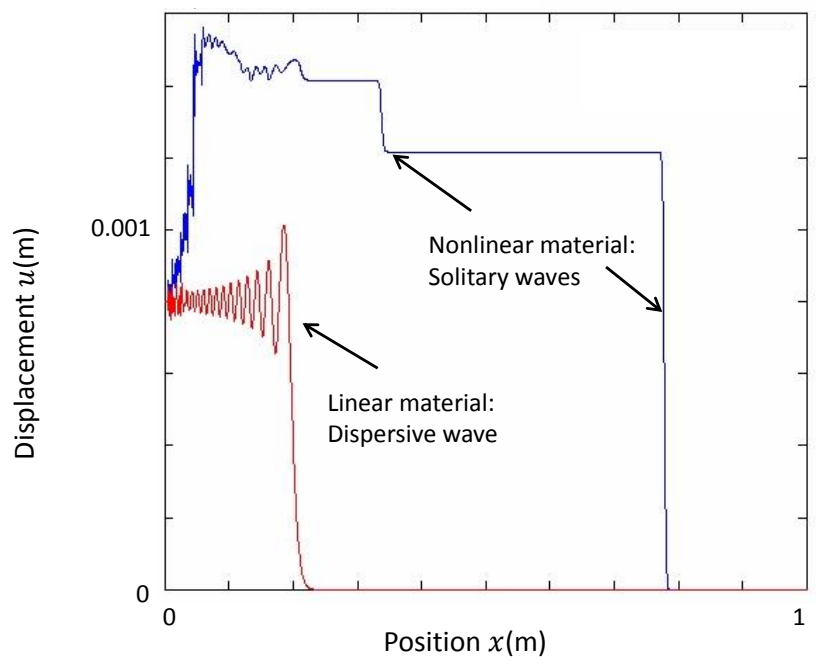

Figure 2: Comparison of wave profiles in linear and nonlinear peridynamic materials in a bar whose left edge is subjected to a step function in displacement at $t=0$. 


\section{Material model}

The material is bond-based and microelastic with pairwise bond force density given by

$$
f(\eta, \xi)=F(s) \operatorname{sgn}(\xi), \quad s=\frac{\eta}{\xi}, \quad 0<|\xi| \leq \delta
$$

where $s$ is the bond strain, $\delta$ is the horizon, $\xi$ is the bond, and $\eta$ is the bond displacement defined by

$$
\eta=u(x+\xi, t)-u(x, t)
$$

where $u$ is the displacement field, $x$ is position, and $t$ is time. The pairwise bond force density defined in (2) has the required antisymmetry

$$
f(-\eta,-\xi)=-f(\eta, \xi)
$$

The function $F$ is defined by

$$
F(s)= \begin{cases}c\left(1-s / s_{0}\right) s & \text { if } s<0 \\ c s & \text { otherwise }\end{cases}
$$

where $c$ and $s_{0}$ are positive constants independent of $\xi$ (Figure 3 ). Thus, the material model is linear in tension but nonlinear in compression. It becomes increasingly stiff as compressive strain is increased. This material model is in some ways similar to the nonlinear spring model that was studied by Fermi, Pasta, and Ulam [5] in their seminal work on nonlinear waves, although the present subject is a continuum rather than a lattice.

The material model (2), (4) has strain energy density $W$ and micropotential $w$ given by

$$
W(x)=\frac{1}{2} \int_{-\delta}^{\delta} w(\eta, \xi) d \xi, \quad w(\eta, \xi)= \begin{cases}\frac{c|\xi| s^{2}}{2}\left(1-\frac{2 s}{3 s_{0}}\right) & \text { if } s<0, \\ \frac{c|\xi| s^{2}}{2} & \text { otherwise }\end{cases}
$$

where $s$ is defined in (2). The pairwise bond force density is related to the micropotential [11] by

$$
f(\eta, \xi)=\frac{\partial w}{\partial \eta}(\eta, \xi)
$$




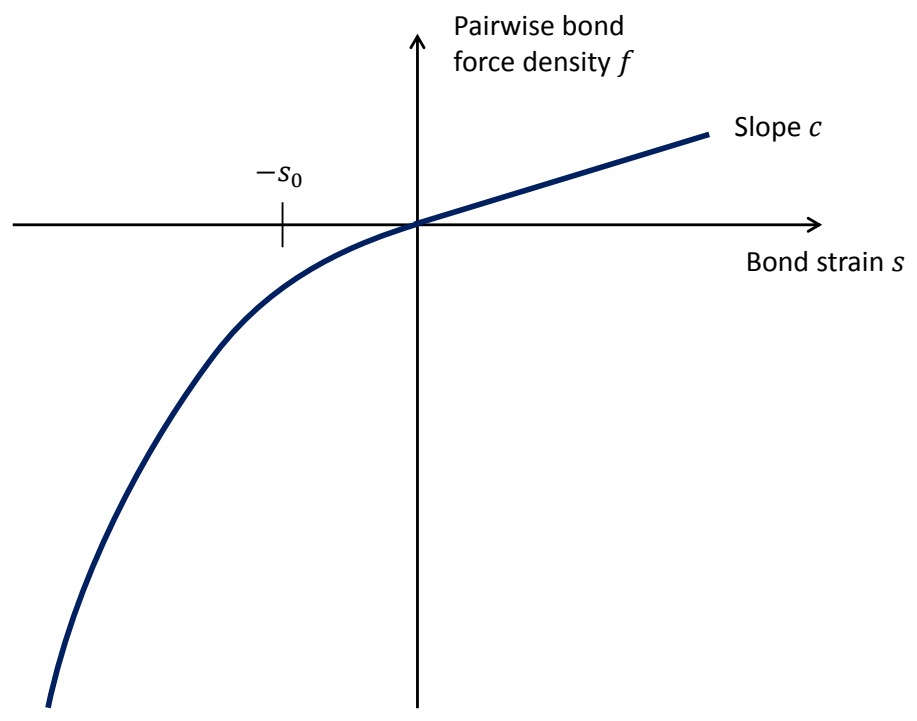

Figure 3: Nonlinear bond-based microelastic material model: pairwise bond force density as a function of bond strain.

The peridynamic equation of motion (1) always conserves total momentum [11]:

$$
\frac{d}{d t} \int_{-\infty}^{\infty} \rho u_{t} d x=\int_{-\infty}^{\infty} b d x
$$

provided that the material model satisfies (3). All microelastic peridynamic materials (that is, materials having a micropotential such that (6) holds) also conserve the total energy:

$$
\frac{d}{d t} \int_{-\infty}^{\infty}\left(\frac{\rho u_{t}^{2}}{2}+W\right) d x=\int_{-\infty}^{\infty} b u_{t} d x
$$




\section{Steady-state wave solution}

Consider a one-dimensional wave pulse moving to the right with constant velocity $V$ through an infinitely long, homogeneous bar. We seek steadystate traveling wave solutions of the form

$$
u(x, t)=U(z)
$$

where $U$ is three times piecewise continuously differentiable, $z$ is the spatial coordinate in the frame of reference of the traveling wave,

$$
z=x-V t
$$

and $V$ is the velocity of the wave (which moves in the $+x$ direction if $V>0$ ). The equation of motion (1) then implies, with $b \equiv 0$,

$$
\rho V^{2} U^{\prime \prime}(z)=\int_{-\delta}^{\delta} f(\eta(z, \xi), \xi) d \xi
$$

where

$$
\eta(z, \xi)=U(z+\xi)-U(z) .
$$

Define the local strain field by

$$
\epsilon(z)=U^{\prime}(z)
$$

The first four terms of a Taylor expansion for $U$ near any $z$ yield

$$
U(z+\xi)=U(z)+U^{\prime}(z) \xi+\frac{U^{\prime \prime}(z) \xi^{2}}{2}+\frac{U^{\prime \prime \prime}(z) \xi^{3}}{6}+\frac{U^{\prime \prime \prime \prime}(z) \xi^{4}}{24}+O\left(\delta^{5}\right)
$$

hence, from (10),

$$
\eta(z, \xi)=U^{\prime}(z) \xi+\frac{U^{\prime \prime}(z) \xi^{2}}{2}+\frac{U^{\prime \prime \prime}(z) \xi^{3}}{6}+\frac{U^{\prime \prime \prime \prime}(z) \xi^{4}}{24}+O\left(\delta^{5}\right) .
$$

Setting

$$
s(z, \xi)=\frac{\eta(z, \xi)}{\xi}
$$

(13) implies

$$
s(z, \xi)=U^{\prime}(z)+\frac{U^{\prime \prime}(z) \xi}{2}+\frac{U^{\prime \prime \prime}(z) \xi^{2}}{6}+\frac{U^{\prime \prime \prime \prime}(z) \xi^{3}}{24}+O\left(\delta^{4}\right)
$$


or equivalently, using (11),

$$
s(z, \xi)=\epsilon(z)+\frac{\epsilon^{\prime}(z) \xi}{2}+\frac{\epsilon^{\prime \prime}(z) \xi^{2}}{6}+\frac{\epsilon^{\prime \prime \prime}(z) \xi^{3}}{24}+O\left(\delta^{4}\right) .
$$

Combining (2), (9), and (11) yields the peridynamic steady-state momentum balance in terms of the local strain field:

$$
\begin{aligned}
\rho V^{2} \epsilon^{\prime}(z) & =\int_{-\delta}^{\delta} F(s(z, \xi)) \operatorname{sgn}(\xi) d \xi \\
& =-\int_{-\delta}^{0} F(s(z, \xi)) d \xi+\int_{0}^{\delta} F(s(z, \xi)) d \xi
\end{aligned}
$$

From (4) and (15), assuming the entire wave is in compression $(s \leq 0)$,

$$
\begin{aligned}
F(s(z, \xi))= & c\left[\epsilon+\frac{\epsilon^{\prime} \xi}{2}+\frac{\epsilon^{\prime \prime} \xi^{2}}{6}+\frac{\epsilon^{\prime \prime \prime} \xi^{3}}{24}+O\left(\delta^{4}\right)\right] \\
& -\frac{c}{s_{0}}\left[\epsilon+\frac{\epsilon^{\prime} \xi}{2}+\frac{\epsilon^{\prime \prime} \xi^{2}}{6}+\frac{\epsilon^{\prime \prime \prime} \xi^{3}}{24}+O\left(\delta^{4}\right)\right]^{2} \\
= & c\left[\epsilon-\frac{\epsilon^{2}}{s_{0}}\right]+c\left[\frac{\epsilon^{\prime}}{2}-\frac{\epsilon \epsilon^{\prime}}{s_{0}}\right] \xi \\
& +c\left[\frac{\epsilon^{\prime \prime}}{6}-\frac{\epsilon \epsilon^{\prime \prime}}{3 s_{0}}-\frac{\epsilon^{\prime 2}}{4 s_{0}}\right] \xi^{2}+c\left[\frac{\epsilon^{\prime \prime \prime}}{24}-\frac{\epsilon^{\prime} \epsilon^{\prime \prime}}{6 s_{0}}-\frac{\epsilon \epsilon^{\prime \prime \prime}}{12 s_{0}}\right] \xi^{3}+O\left(\delta^{4}\right) .
\end{aligned}
$$

After substituting this expression into the integrands in (16) and evaluating the integrals, only the terms involving odd powers of $\xi$ survive, with the result

$$
\frac{\rho V^{2}}{c} \epsilon^{\prime}=\left[\frac{\epsilon^{\prime}}{2}-\frac{\epsilon \epsilon^{\prime}}{s_{0}}\right] \delta^{2}+\left[\frac{\epsilon^{\prime \prime \prime}}{24}-\frac{\epsilon^{\prime} \epsilon^{\prime \prime}}{6 s_{0}}-\frac{\epsilon \epsilon^{\prime \prime \prime}}{12 s_{0}}\right] \frac{\delta^{4}}{2}+O\left(\delta^{6}\right),
$$

or, after dropping the high order term,

$$
\left[\frac{\rho V^{2}}{c}-\frac{\delta^{2}}{2}\right] \epsilon^{\prime}=\left[-\frac{\epsilon \epsilon^{\prime}}{s_{0}}\right] \delta^{2}+\left[\frac{\epsilon^{\prime \prime \prime}}{24}-\frac{\epsilon^{\prime} \epsilon^{\prime \prime}}{6 s_{0}}-\frac{\epsilon \epsilon^{\prime \prime \prime}}{12 s_{0}}\right] \frac{\delta^{4}}{2} .
$$

The calibration of $c$ is accomplished by assuming a small, uniform strain $\gamma$ in the bar under uniaxial stress. The Young's modulus of the bar (for small strains) is $E$, which must be independent of $\delta$. By summing the force 
densities in all the bonds that cross some given point in the bar, and using (4), one finds that the stress is given by

$$
\sigma=\int_{0}^{\delta} F(\gamma) \xi d \xi=\int_{0}^{\delta} c\left(1-\frac{\gamma}{s_{0}}\right) \gamma \xi d \xi=\frac{c \delta^{2} \gamma}{2}-\frac{c \delta^{2} \gamma^{2}}{2 s_{0}}
$$

Since, for linear elastic response, $\sigma=E \gamma$, it follows from (18) that

$$
c=\frac{2 E}{\delta^{2}}
$$

From (17) and (19),

$$
\left(\frac{\rho V^{2}}{E}-1\right) \epsilon^{\prime}=\left[-\frac{2 \epsilon \epsilon^{\prime}}{s_{0}}\right]+\left[\frac{\epsilon^{\prime \prime \prime}}{24}-\frac{\epsilon^{\prime} \epsilon^{\prime \prime}}{6 s_{0}}-\frac{\epsilon \epsilon^{\prime \prime \prime}}{12 s_{0}}\right] \delta^{2} .
$$

This nonlinear third order ODE is the key equation in our approximate solution method. The objective is to obtain solutions to (20) such that

$$
\epsilon( \pm \infty)=\epsilon^{\prime}( \pm \infty)=\epsilon^{\prime \prime}( \pm \infty)=0, \quad \int_{-\infty}^{\infty} \epsilon(z) d z=-\Delta U
$$

where $\Delta U$ is the displacement amplitude, the prescribed total change in displacement across the wave.

As an aside, it can be concluded from (20) that formally, in the limiting case of small wave amplitude,

$$
|\epsilon| \ll s_{0} \quad \Longrightarrow \quad\left(\frac{\rho V^{2}}{E}-1\right) \epsilon^{\prime}=\frac{\delta^{2}}{24} \epsilon^{\prime \prime \prime},
$$

which is simply a form of the linear wave equation for steady-state waves, provided that the term in parentheses is negative. Hence, for a small-amplitude wave train with wave number $\kappa$,

$$
\epsilon(z)=\epsilon_{0} \cos \kappa z, \quad\left|\epsilon_{0}\right| \ll s_{0},
$$

and therefore the velocity of the linear wave train is found from (21) to be

$$
V=\sqrt{\frac{E}{\rho}\left(1-\frac{\delta^{2} \kappa^{2}}{24}\right)}
$$




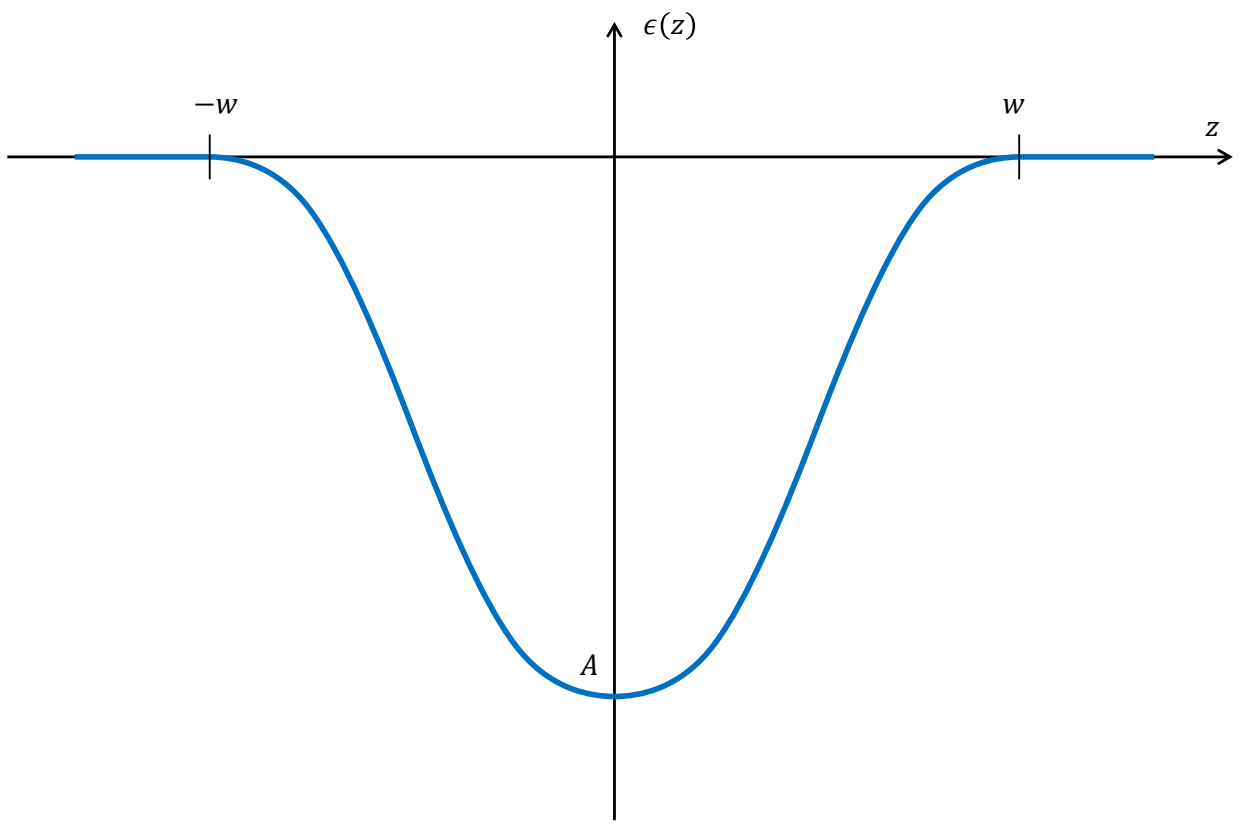

Figure 4: Assumed form of the strain profile $\epsilon(z)$ within a solitary wave.

This shows that the classical linear wave velocity $\sqrt{E / \rho}$ is an upper bound in the dispersion relation. The classical velocity is approached for either small horizon $(\delta \rightarrow 0)$ or long waves $(\kappa \rightarrow 0)$. The conclusion that finitewavelength waves are slower than the classical wave velocity is consistent with all previous analysis of waves in peridynamic media, for example [11].

Returning to the large amplitude case, we will attempt to find a displacement field satisfying (20) without dropping any of the nonlinear terms. To do this, assume as an ansatz that

$$
\epsilon(z)= \begin{cases}A \cos ^{2} k z & \text { if }|k z| \leq \pi / 2 \\ 0 & \text { otherwise }\end{cases}
$$

where $A$ and $k$ are constants to be determined and $A<0$ (Figure 4).

The combinations of derivatives that appear in (20) are found by differ- 
entiation of (22) to be given by, for $|k z| \leq \pi / 2$,

$$
\begin{aligned}
\epsilon^{\prime} & =-2 A k \cos k z \sin k z, \\
\epsilon^{\prime \prime} & =2 A k^{2}\left(\sin ^{2} k z-\cos ^{2} k z\right)=2 A k^{2}\left(1-2 \cos ^{2} k z\right), \\
\epsilon^{\prime \prime \prime} & =8 A k^{3} \cos k z \sin k z, \\
\epsilon \epsilon^{\prime} & =-2 A^{2} k \cos ^{3} k z \sin k z, \\
\epsilon^{\prime} \epsilon^{\prime \prime} & =8 A^{2} k^{3} \cos ^{3} k z \sin k z-4 A^{2} k^{3} \cos k z \sin k z, \\
\epsilon \epsilon^{\prime \prime \prime} & =8 A^{2} k^{3} \cos ^{3} k z \sin k z .
\end{aligned}
$$

Substituting these expresssions into (20) yields

$$
\begin{aligned}
& \left(\frac{\rho V^{2}}{E}-1\right)(-2 A k \cos k z \sin k z)=\frac{2}{s_{0}}\left(2 A^{2} k \cos ^{3} k z \sin k z\right) \\
& \quad+\frac{\delta^{2}}{24}\left(8 A k^{3} \cos k z \sin k z\right)-\frac{\delta^{2}}{6 s_{0}}\left(8 A^{2} k^{3} \cos ^{3} k z \sin k z-4 A^{2} k^{3} \cos k z \sin k z\right) \\
& \quad-\frac{\delta^{2}}{12 s_{0}}\left(8 A^{2} k^{3} \cos ^{3} k z \sin k z\right) .
\end{aligned}
$$

Rearranging this gives

$0=\frac{2 A^{2} k}{s_{0}}\left(2-k^{2} \delta^{2}\right) \cos ^{3} k z \sin k z+A k\left[2\left(\frac{\rho V^{2}}{E}-1\right)+\frac{k^{2} \delta^{2}}{3}+\frac{2 A k^{2} \delta^{2}}{3 s_{0}}\right] \cos k z \sin k z$.

Since (24) must hold for all $z$, the coefficient of $\cos ^{3} k z \sin k z$ must vanish, leading to

$$
k=\frac{\sqrt{2}}{\delta} .
$$

Similarly, the coefficient of $\cos k z \sin k z$ in (24) must vanish, which, using (25), implies

$$
V= \pm \sqrt{\frac{2 E}{3 \rho}\left(1-\frac{A}{s_{0}}\right)}
$$

(recall that $A<0$ by assumption). Let $w$ denote the half-width of the pulse; hence the form of (22) implies

$$
k=\frac{\pi}{2 w}
$$

and

$$
\epsilon(z)=A \cos ^{2}\left(\frac{\pi z}{2 w}\right), \quad|z| \leq w
$$


From (25) and (27),

$$
w=\frac{\pi}{2 k}=\frac{\pi \delta}{\sqrt{8}} .
$$

Assume that the wave moves to the right $(V>0)$ and that the displacement amplitude $\Delta U>0$ is given. Since $\epsilon=U^{\prime}$,

$$
\int_{-w}^{w} \epsilon(z) d z=-\Delta U .
$$

Evaluating the integral in (30) using (28) leads to

$$
\Delta U=-A w .
$$

From (29) and (31),

$$
A=-\frac{\Delta U}{w}=-\frac{\sqrt{8} \Delta U}{\pi \delta} .
$$

Combining (28), (29), and (32) leads to the following expression for the local strain profile within the wave in terms of the displacement amplitude $\Delta U$ :

$$
\epsilon(z)= \begin{cases}-\frac{\sqrt{8} \Delta U}{\pi \delta} \cos ^{2}\left(\frac{\sqrt{2} z}{\delta}\right) & \text { if }|z| \leq w \\ 0, & \text { otherwise }\end{cases}
$$

where $w$ is given by the second of (29). The fact that $\epsilon^{\prime \prime}(z)$ computed from (33) is discontinuous at $z= \pm w$ does not affect the validity of the solution, because $\epsilon^{\prime \prime}$ only appears in (20) in the combination $\epsilon^{\prime} \epsilon^{\prime \prime}$. Since

$$
\epsilon( \pm w)=\epsilon^{\prime}( \pm w)=\epsilon^{\prime \prime \prime}( \pm w)=0
$$

(33) satisfies the ODE (20) everywhere, including $z= \pm w$.

In summary, for a wave pulse with a given displacement amplitude $\Delta U$, the ansatz (22) is confirmed in which the strain amplitude $A$ is given by (32). The half-width of the pulse $w$ is independent of $\Delta U$ and is given by (29). The velocity of the pulse is given by (26), which can be rewritten using (32) as

$$
V= \pm \sqrt{\frac{2 E}{3 \rho}\left(1+\frac{\sqrt{8} \Delta U}{\pi \delta s_{0}}\right)}
$$


Since the solitary wave in (33) has compact support $[-w, w]$, it is similar to a compacton [10]. However, it is not a soliton because, as shown in Section 5.3 below, waves create a small dispersive wake when they pass through each other. Strictly speaking, solitons are a special type of solitary wave that do not interact with each other at all, although sometimes these terms are used interchangeably.

The momentum $P$ of the solitary wave can be defined as the momentum of all the material within it. To compute the material velocity, from (7), (8), and (11),

$$
u_{t}=-V \epsilon
$$

Then from (22) and (27),

$$
P=\int_{-\infty}^{\infty} \rho u_{t} d x=\int_{-\infty}^{\infty} \rho(-V \epsilon) d z=-\rho V A \int_{-w}^{w} \cos ^{2}\left(\frac{\pi z}{2 w}\right) d z=-\rho V A w
$$

(recall that $A<0$ ). Proceeding similarly for the kinetic energy of the wave $K$

$$
K=\frac{1}{2} \int_{-\infty}^{\infty} \rho u_{t}^{2} d x=\frac{\rho V^{2} A^{2}}{2} \int_{-w}^{w} \cos ^{4}\left(\frac{\pi z}{2 w}\right) d z=\frac{3 \rho V^{2} A^{2} w}{8}
$$

\section{Examples and numerical simulations}

This section describes comparison between results of the approximate analytical model derived above and computational simulations. The numerical method is described in [12].

\subsection{Solitary wave shape and velocity}

The strain profile within a wave predicted by (33) is compared here with the result of a one-dimensional computational simulation. A solitary wave moves to the right with displacement amplitude $\Delta U=0.000162 \mathrm{~m}$. The material has Young's modulus $E=1.0 \mathrm{E} 9 \mathrm{~Pa}$ and density $\rho=1000 \mathrm{~kg} / \mathrm{m}^{3}$, with the nonlinear material parameter $s_{0}=0.01$. The horizon is $\delta=0.0061 \mathrm{~m}$. The speed of waves in the local linear model is $V_{\text {local }}=\sqrt{E / \rho}=1000 \mathrm{~m} / \mathrm{s}$. The numerical model uses a grid spacing of $0.0005 \mathrm{~m}$, resulting in a resolution of about 12 nodes through the horizon. Increasing the number of nodes by a factor of 2 does not significantly change the results. 
The parameters for the analytical model (28) are found as follows. From (29) and (32),

$$
w=0.00678 \mathrm{~m}, \quad A=-0.0239 .
$$

From (34), $V=1503 \mathrm{~m} / \mathrm{s}$. Clearly $V>V_{\text {local }}$.

The computational simulation of this problem predicts a propagation velocity of the solitary wave of $1600 \mathrm{~m} / \mathrm{s}$ and a peak strain of $A=-0.0248$. The disagreement between the analytical model parameters and the computed values is mainly due to the neglect of high order terms in the derivation of the ODE (20). Figures 5 and 6 compare the displacement and local strain fields within the wave for the analytical model and the computational simulation.

Similar calculations were performed over a range of displacement amplitude. The wave velocities from (34) are compared with the simulated values in Figure 7 . The difference is about $10 \%$ through the range. For small values of displacement amplitude in the range

$$
\Delta U<\frac{\pi \delta s_{0}}{\sqrt{32}}=3.39 \mathrm{E}-5 \mathrm{~m},
$$

the wave velocity from (34) is less than $V_{\text {local }}$. In these cases, the numerical model predicts linear dispersive waves similar to those seen in the example in Figure 2. Under these conditions, the solitary wave solution does not apply because the assumption of a steady-state (constant shape) wave does not hold.

The similarity between the numerical simulations and the approximate analysis justifies the general approach of approximating the integral equation with a finite number of terms, as in (12). The fact that the wave is continuous and has a persistent, finite thickness means that it can be approximated by a truncated Taylor expansion with a remainder term.

\subsection{Relation to shock waves}

A solitary wave leaves conditions unchanged in the material that it passes through, except for a rigid translation $\Delta U$. If the rear half of the pulse is excluded, that is, if (22) is replaced by

$$
\epsilon_{\star}(z)= \begin{cases}A \cos ^{2} k z & \text { if } 0 \leq k z \leq \pi / 2, \\ A & \text { otherwise }\end{cases}
$$




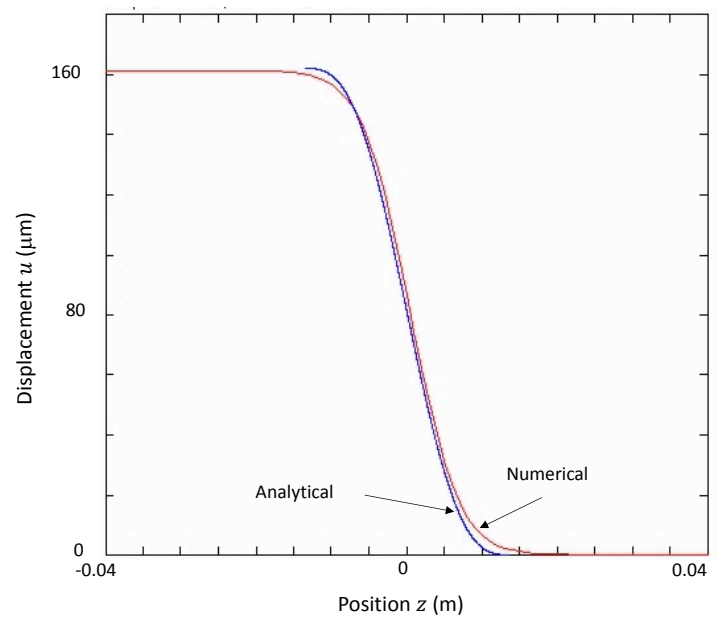

Figure 5: Displacement as a function of position in the example problem.

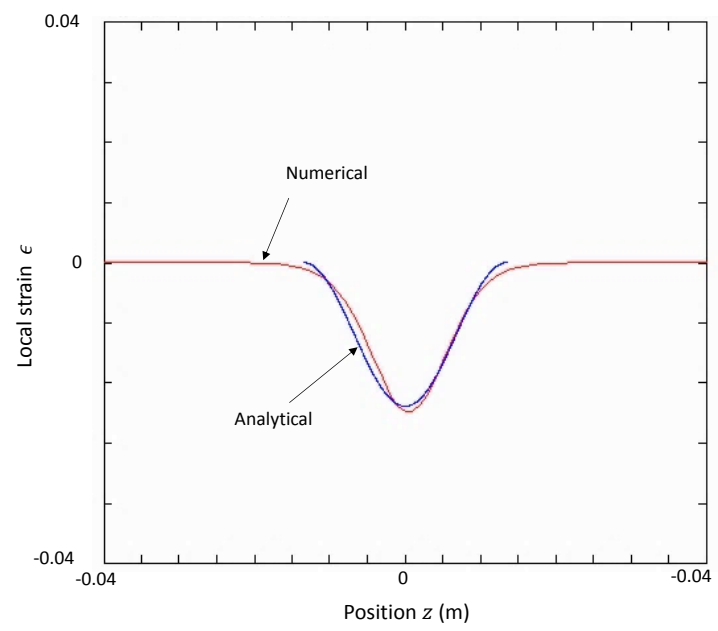

Figure 6: Local strain $\epsilon=U^{\prime}$ as a function of position in the example problem. 


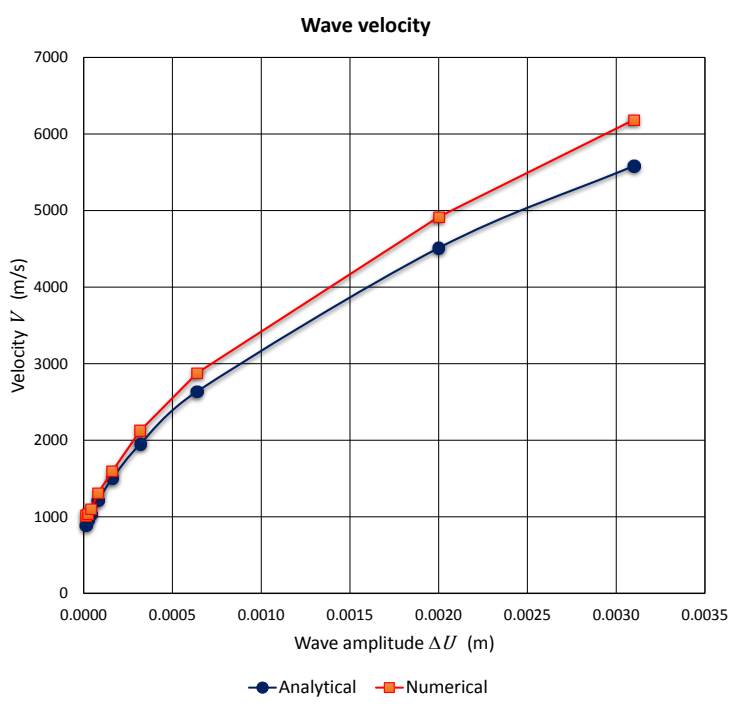

Figure 7: Wave velocity as a function of displacement amplitude from the approximate analytical expression (34) and from computational simulations. The linear wave velocity for a material with the same $E$ and $\rho$ is $1000 \mathrm{~m} / \mathrm{s}$. 
this local strain field is still a solution to (20). At first glance, this would appear to be a satisfactory representation of a shock wave for this material. However, it fails to satisfy the Rankine-Hugoniot jump condition, so it is an inadmissible solution. (Recall that (9) and therefore (20) are based on the assumption of a steady-state wave. This assumption fails to hold in the present case of a shock rather than a solitary wave.)

The net effect is that shock wave solutions of the form (37) are never observed in the computational simulations. Instead, the shock wave decomposes into many solitary waves that form a wavetrain, as illustrated in Figure 8. This figure shows the velocity field induced by a constant velocity boundary condition applied to the left end of a bar. The spontaneous decomposition of a smooth velocity field into multiple solitary waves occurs in many other contexts and is a characteristic feature of nonlinear waves [20]. The failure of nonlinear elastic materials to reproduce steady shock waves is characteristic of the local theory as well as the peridynamic theory. It can be remedied by including dissipative terms in the material model (thereby making the model inelastic).

Equilibrium phase boundaries are similar to shock waves in that they are often modeled within the local theory as discontinuities in strain that obey jump conditions [4, 1]. Dayal and Bhattacharya investigated peridynamic models for phase boundaries [3]. The peridynamic approach yields phase boundaries with finite thickness. In equilibrium, the internal structure of such a phase boundary is well-defined and stable. In dynamics, a moving phase boundary arising from an elastic peridynamic material model releases short-wavelength waves similar to those shown in Figure 8.

\subsection{Interaction between solitary waves}

Figure 9 shows a numerical simulation of the collision between two peridynamic solitary waves. The material model and parameters are the same as in Section 5.1. The variable on the vertical axis in the plots is the local strain $u_{x}=\epsilon$. In the left plot, the larger wave is about to overtake the smaller, slower wave. The interaction releases some small dispersive reflections that are in the linear regime of the material response. These small reflections, which are sometimes called a dispersive tail, are seen in solitary waves in various physical systems [2]. Aside from these reflections, the two solitary waves emerge from the collision with little change in their peak strain or velocity, as shown in the plot on the right. The center plot shows a time 


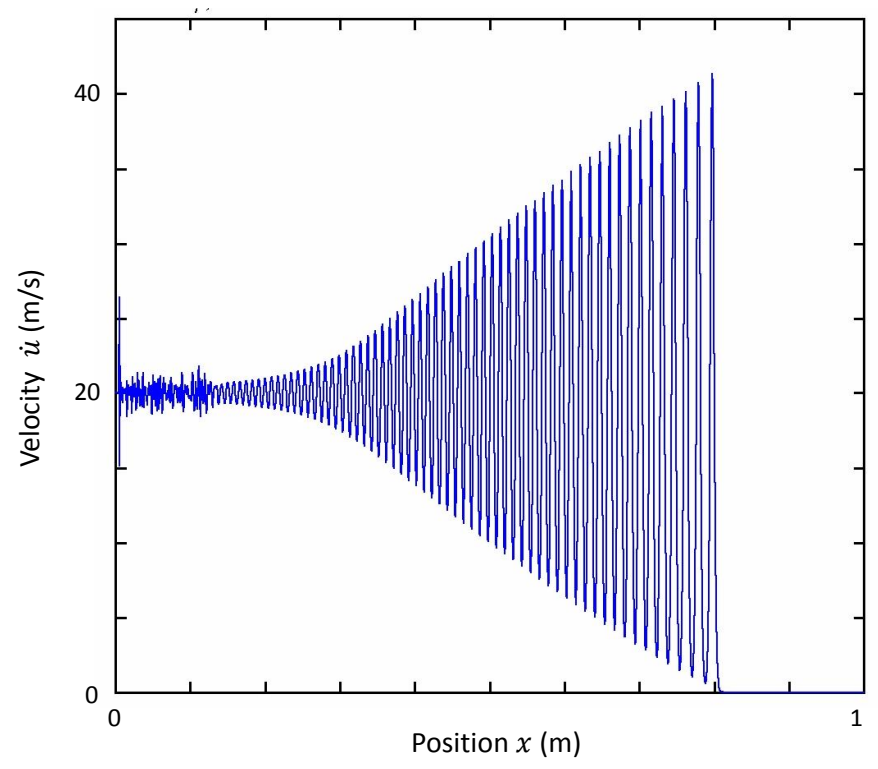

Figure 8: Train of solitary waves propagating to the right generated by a constant velocity of $20 \mathrm{~m} / \mathrm{s}$ applied to the left boundary. 

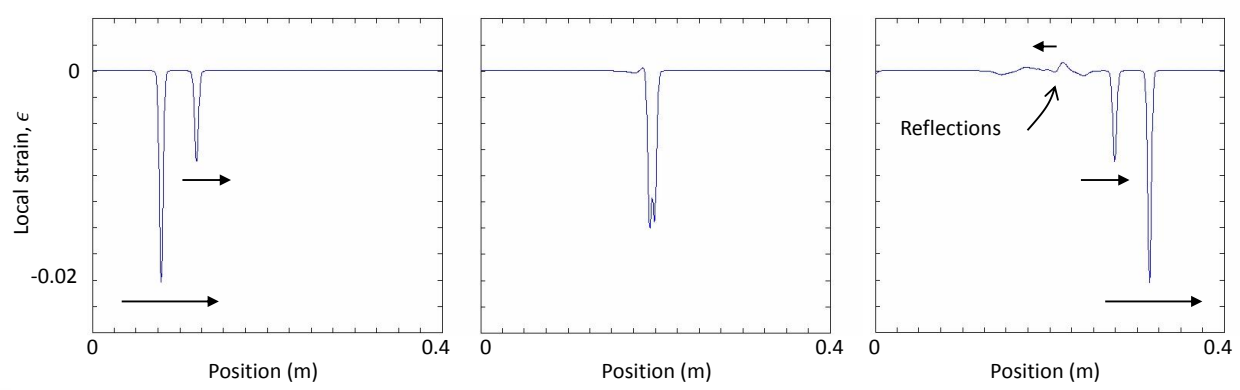

Figure 9: Local strain as a function of position as a larger solitary wave overtakes a smaller one. Left: before the collision. Center: during the collision. Right: after the collision. Horizontal arrows indicate the wave velocities.

when the two waves are almost exactly on top of each other. The peak strain in the combined wave is much less than the sum of the peak strains in the incident waves. This observation suggests that the interaction process is fundamentally different from the superposition of linear waves, which also permits waves to pass through each other. In the linear case, the peak strain at the time of collision would equal the sum of the peak strains in the two incident waves.

\section{Discussion}

The main result of the present paper is that large amplitude nonlinear waves in a peridynamic medium can propagate as solitary waves that move without 
dispersion at speeds much greater than the linear wave velocity. The thickness of the wave depends only on the horizon, although the wave velocity depends strongly on the amplitude. Wavetrains consisting of many solitary waves can form. These wavetrains allow the material to accomodate sudden changes in velocity at the boundary, since shock waves are not possible in the absence of dissipative terms in the material model or artificial viscosity. Peridynamic solitary waves result from the equilibrium between nonlinearity in the material model, which tends to make the pulse narrower, and the naturally dispersive character of the model due to nonlocality. The peridynamic solitary waves exhibit many of the same characteristics observed in solitary waves in many other physical systems, including weak interactions between waves that collide or overtake each other.

The behavior of short-wavelength waves in linear elastic peridynamic materials is related to phenomena such as the emergence of discontinuities in displacement [17]. The dispersion curves of such materials dictate that shortwavelength waves have lower wave speeds than longer waves. This feature leads to the evolution of persistent discontinuities in displacement in initial value problems with discontinuous initial data. In the present case of solitary waves, the solitary wave velocity $V$ is always larger than the velocity of any linear wave, regardless of wavelength. As shown in Figure 2, small amplitude linear waves are left in the wake of the faster-moving soliton. Because of their fundamentally nonlinear nature, the internal structure of solitary waves cannot be understood in terms of the Fourier spectrum of linear waves.

The peridynamic treatment of solitary waves does not involve any alteration of the field equations. The solitary waves arise solely from the choice of a particular nonlinear material model. This is in contrast to classical treatments of solitary waves that rely on special versions of the field equations. Usually these are third or higher order differential equations that are not derivable from the standard equations of solid mechanics.

It is of interest to compare the present model with the Korteweg-de Vries $(\mathrm{KdV})$ equation:

$$
\phi_{t}+a_{0} \phi_{x}+a_{1} \phi \phi_{x}+a_{2} \phi_{x x x}=0
$$

where $a_{0}, a_{1}, a_{2}$ are constants. The steady wave assumption leads to the following ODE:

$$
\left(a_{0}-C\right) \epsilon^{\prime}+a_{1} \epsilon \epsilon^{\prime}+a_{2} \epsilon^{\prime \prime \prime}=0, \quad \phi(x, t)=\epsilon(x-C t) .
$$

Compare this with the ODE (20) from the approximate peridynamic analysis 
above:

$$
\left(\frac{\rho V^{2}}{E}-1\right) \epsilon^{\prime}+\frac{2}{s_{0}} \epsilon \epsilon^{\prime}+\left[-\frac{\epsilon^{\prime \prime \prime}}{24}+\frac{\epsilon^{\prime} \epsilon^{\prime \prime}}{6 s_{0}}+\frac{\epsilon \epsilon^{\prime \prime \prime}}{12 s_{0}}\right] \delta^{2}=0 .
$$

The last two equations share some terms in common: $\epsilon^{\prime}, \epsilon \epsilon^{\prime}$, and $\epsilon^{\prime \prime \prime}$. However, the peridynamic equation has additional nonlinear terms that lead to solutions that differ in significant ways from solitary waves predicted by the $\mathrm{KdV}$ equation. In particular, in contrast to the $\mathrm{KdV}$ waves, the peridynamic solitary waves are compact, as discussed in Section 4. Detailed discussion of solutions to the KdV equation can be found in [19].

\section{Acknowledgment}

Sandia National Laboratories is a multi-program laboratory operated by Sandia Corporation, a wholly owned subsidiary of Lockheed Martin Corporation, for the U.S. Department of Energy's National Nuclear Security Administration under contract DE-AC04-94AL85000.

\section{References}

[1] R. Abeyaratne and J. K. Knowles. Equilibrium shocks in plane deformations of incompressible elastic materials. Journal of elasticity, 22(23):63-80, 1989.

[2] J. L. Bona, W. Pritchard, and L. R. Scott. Solitary-wave interaction. Physics of Fluids (1958-1988), 23:438-441, 1980.

[3] K. Dayal and K. Bhattacharya. Kinetics of phase transformations in the peridynamic formulation of continuum mechanics. Journal of the Mechanics and Physics of Solids, 54:1811-1842, 2006.

[4] J. Ericksen. Equilibrium of bars. Journal of elasticity, 5(3):191-201, 1975.

[5] E. Fermi, J. Pasta, and S. Ulam. Studies of non linear problems. Technical Report LA-1940, Los Alamos National Laboratory, May 1955.

[6] M. A. Hasan and S. Nemat-Nasser. Universal relations for solitary waves in granular crystals under shocks with finite rise and decay times. Physical Review E, 93(4):042905, 2016. 
[7] W. Hu, Y. Wang, J. Yu, C.-F. Yen, and F. Bobaru. Impact damage on a thin glass plate with a thin polycarbonate backing. International Journal of Impact Engineering, 62:152-165, 2013.

[8] E. Madenci and E. Oterkus. Peridynamic Theory and Its Applications. Springer, New York, 2013.

[9] V. Nesterenko. Propagation of nonlinear compression pulses in granular media. Journal of Applied Mechanics and Technical Physics, 24:733-743, 1983.

[10] P. Rosenau and J. M. Hyman. Compactons: solitons with finite wavelength. Physical Review Letters, 70:564-567, 1993.

[11] S. A. Silling. Reformulation of elasticity theory for discontinuities and long-range forces. Journal of the Mechanics and Physics of Solids, 48:175-209, 2000.

[12] S. A. Silling and E. Askari. A meshfree method based on the peridynamic model of solid mechanics. Computers and Structures, 83:1526-1535, 2005.

[13] S. A. Silling and F. Bobaru. Peridynamic modeling of membranes and fibers. International Journal of Non-Linear Mechanics, 40:395-409, 2005 .

[14] S. A. Silling, M. Epton, O. Weckner, J. Xu, and E. Askari. Peridynamic states and constitutive modeling. Journal of Elasticity, 88:151$184,2007$.

[15] S. A. Silling and R. B. Lehoucq. The peridynamic theory of solid mechanics. Advances in Applied Mechanics, 44:73-166, 2010.

[16] M. Toda. Waves in nonlinear lattice. Progress of Theoretical Physics Supplement, 45:174-200, 1970.

[17] O. Weckner and E. Emmrich. Numerical simulation of the dynamics of a nonlocal, inhomogeneous, infinite bar. Journal of Computational and Applied Mechanics, 6:311-319, 2005. 
[18] O. Weckner and S. A. Silling. Determination of the constitutive model in peridynamics from experimental dispersion data. International Journal of Multiscale Computational Engineering, 9:623-634, 2011.

[19] G. B. Whitham. Linear and nonlinear waves. John Wiley \& Sons, 1974.

[20] N. J. Zabusky and M. D. Kruskal. Interaction of solitons in a collisionless plasma and the recurrence of initial states. Physical Review Letters, 15:240-243, 1965. 